\title{
Modelos de Investimento: Metodologia e Resultados
}

\author{
Investment Models: Methodology and Results
}

ELTON EUSTÁQUIO CASAGRANDE*

\begin{abstract}
RESUMO: Este artigo fornece uma visão geral dos estudos empíricos sobre padrões de financiamento corporativo e comportamento de investimento da empresa. Em um mundo sem atrito, o teorema de Modigliani-Miller é válido, assim como a teoria do investimento neoclássico. No entanto, na presença de imperfeições resultantes de assimetrias de informação, o financiamento interno costuma ser menos oneroso do que o financiamento externo. $\mathrm{O}$ objetivo deste artigo é mostrar como os estudos empíricos examinaram o papel das restrições financeiras e o comportamento do investimento da empresa.

PALAVRAS-CHAVES: Investimento; assimetria; liquidez; financiamento.
\end{abstract}

ABSTRACT: This paper provides an overview of the empirical studies on corporate financing patterns and firm's investment behavior. In a frictionless world the Modigliani-Miller theorem holds as well as the neoclassical investment theory. However, in presence of imperfections resulting from information asymmetries internal finance is often less costly than external finance. The goal in this article is to show how empirical studies have examined the role of financial constraints and firm's investment behavior.

KEYWORDS: Investment; asymmetry; liquidity; financing.

JEL Classification: G31.

\section{APRESENTAÇÃO}

A decisão de investimento constitui uma das mais importantes decisões econômicas, se for considerado que ela define, em nível agregado, o desempenho da economia. É também fonte da acumulação de capital e, portanto, a maior determinante da capacidade da economia a longo prazo. Finalmente, é a componente mais instável da demanda agregada e natural responsável pelas variações na renda e no emprego. Porém, não é explicada adequadamente pelos modelos convencionais do

\footnotetext{
* Professor do Departamento de Economia da Unesp — Campus Araraquara - SP. E-mail: elton@fclar. unesp.br; Orcid: 0000-0003-4523-6802.
} 
investimento, porque não capturam os efeitos da incerteza sobre essa decisão. Pindyck (1991) observa que a despeito dessa importância, o comportamento do investimento de firmas, indústrias e de países permanece pouco entendido.

Neste artigo tentamos explicar por que a afirmação de Pindyck (1991), embora tenha feito completo sentido no inicio da década, não pode ser repetida com a mesma contundência. Para realizar essa tarefa, resenharemos diversas contribuições sobre a teoria do investimento, de modo que configurem uma resenha da própria teoria. Nessa análise das teorias estamos interessados nas principais contribuições que tratam do comportamento do investimento de firmas, uma tarefa que implica em recuperar o modelo do acelerador e suas versões posteriores, utilizadas para explicar sob diferentes hipóteses, o gasto de investimento ${ }^{1}$.

Este artigo está estruturado da seguinte forma: a segunda seção trata das modificações feitas no modelo do acelerador pela versão neoclássica; a terceira seção resenha os principais modelos empíricos do investimento que testaram a hipótese da assimetria de informações. Demos destaque à metodologia desses estudos. Finalmente, na quarta seção, discutimos a afirmação feita Robert Pindyck sobre a capacidade explicativa dos modelos empíricos do investimento.

\section{O MODELO NEOCLÁSSICO}

O modelo genérico, segundo a forma de apresentação proposta por Chirinko (1993), permite mostrar a evolução da teoria do investimento. O autor, estabelecendo um Benchmark Model, escreve a função demanda por capital como:

$$
\mathrm{K}^{*}=\mathrm{f}[\text { preços, quantidades, choques] }
$$

A demanda por capital é determinada pela igualdade entre os benefícios marginais esperados e o custo de uma unidade adicional de capital. A demanda por investimento inclui um aspecto dinâmico na função, denominado acelerador. O princípio do acelerador faz o investimento ser uma proporção linear das mudanças no produto: a partir de um incremento na taxa capital-produto, é simples calcular o investimento necessário para atender a uma dada meta de crescimento do produto.

A partir do acelerador, Hall \& Jorgenson (1967) e seus colaboradores formularam a teoria neoclássica do investimento. Em termos práticos, o modelo neoclássico parte da equação (1), utilizando-se de uma estrutura rigorosa para identificar as variáveis explicativas do investimento, especialmente os efeitos dos preços relativos. A firma encontra instantaneamente o estoque ótimo de capital, $\mathrm{K}^{*}$, o qual

\footnotetext{
${ }^{1}$ É útil fazer a distinção entre abordagens que relacionam a taxa de investimento com uma dada variável macroeconômica e outras que lidam com os microfundamentos da decisão de investimento, em que a taxa de lucro e de juros são as duas variáveis relevantes. Segundo Bresser-Pereira (1993), Kalecki (1952), Clark (1917) e Chenery (1952) apud Bresser-Pereira (1993) são exemplos da primeira abordagem do investimento. Na segunda, estão a teoria clássica que enfatiza a taxa de lucro como principal motivo da decisão, enquanto a teoria neoclássica enfatiza a taxa de juros.
} 
maximiza seus lucros (otimização estática). O estoque ótimo de capital depende do preço dos bens de capital, da taxa de juros e da depreciação. Dadas as condições técnicas, uma firma investirá em uma nova planta ou na compra de máquinas e equipamentos, se o valor de um dado projeto exceder o custo de capital, líquido de impostos ${ }^{2}$. Nos termos acima, na comparação do custo de capital com a produtiviFdade marginal do novo investimento, o retorno previsto deve ser suficiente para compensar os acionistas pelo custo de oportunidade. Fatores que elevam a taxa de juros, afetando o custo de capital e o volume de investimento são, portanto, concorrentes ao investimento.

A formulação neoclássica apresenta uma equação para testar os determinantes do investimento e, especialmente, os efeitos dos preços relativos, introduzidos na Teoria Geral, em que os custos e benefícios de adquirir capital eram relacionados à eficiência marginal do capital e da taxa de juros (Chirinko, 1993):

$$
\text { It }=\delta \mathrm{Kt}-1+\sum_{\mathrm{j}-0}^{\mathrm{J}} \alpha \beta \mathrm{j} \Delta\left(\mathrm{Y}_{\mathrm{t}-\mathrm{j}} \mathrm{C}_{\mathrm{t} \cdot \mathrm{j}}^{-\sigma}\right)+\mu_{\mathrm{t}}
$$

onde It $=$ investimento total; $\delta=$ depreciação; $\sigma=$ elasticidade de substituição entre capital e trabalho; $\alpha=$ parâmetro de distribuição; $\beta=$ defasagem de entrega do equipamento; $\mathrm{Y}=$ variável quantitativa (vendas); $\mathrm{Ct}=$ custo do capital; $\mu=$ erro $^{3}$.

O modelo neoclássico considera os fundos externos como perfeitos substitutos aos fundos internos das firmas. A aceitação do teorema Modigliani \& Miller (1958 e 1961) faz o gasto de investimento ser explicado pelas variáveis reais, como os preços e a tecnologia, e trata com absoluta independência as relações com o mercado de capitais e de crédito. Com essa formulação, Jorgenson provou que o coeficiente da variável vendas é dominante e estatisticamente relevante para explicar o comportamento do investimento. As firmas escolhem o quanto produzir sob a noção de mercados em concorrência perfeita, vendendo a oferta que criam. As expectativas de vendas das firmas têm, portanto, um importante impacto sobre o gasto de investimento. Em termos econométricos, essa variável predomina sobre outras que possam vir a ser incluídas na equação, sendo assim subjacente ao modelo proposto por Clark (1917) apud Chirinko (1993).

Segundo Fazzari \& Petersen (1993), as versões derivadas do acelerador, como da teoria neoclássica, têm sido utilizadas com excelentes resultados, porém os fortes efeitos do acelerador têm dificultado uma avaliação mais realista do próprio modelo. Na versão neoclássica o custo de capital afeta o investimento somente por meio de variáveis que também incluem as vendas. Não se podem separar os efeitos das vendas e do custo de capital sobre o investimento, e tratá-las independentemente do ponto de vista da política econômica. $\mathrm{Na}$ expectativa de um volume de vendas

\footnotetext{
${ }^{2} \mathrm{O}$ custo de capital define-se como o custo de oportunidade de um investimento, medido pela taxa de juros ajustada tanto ao risco, quanto à inflação futura.

${ }^{3}$ Esta equação contém algumas modificações desde a elaboração original de Clark (1917) apud Chirinko (1993, p.1878-79).
} 
maior, a firma ajusta seu estoque de capital sempre que a taxa de retorno do investimento seja, no mínimo, igual ao custo de capital, representado pela taxa de juros.

Dois aspectos em relação à teoria neoclássica precisam ser observados. $\mathrm{O}$ primeiro é a predominância da teoria neoclássica sobre outras contribuições que não tiveram êxito ao tentar demonstrar a importância das variáveis financeiras sobre as reais para a decisão de investimento. Ao manterem as noções de mercados em concorrência perfeita e firma representativa, os estudos limitaram sua própria capacidade de verificar a importância das variáveis financeiras enquanto determinante do investimento entre firmas com diferentes características. Entre os estudos rejeitados pela abordagem neoclássica e analisados em Jorgenson (1971) temos: Meyer \& Kuh (1957), Evans (1967), Eisner (1963), Meyer \& Glauber (1964) e Anderson (1964). Segundo os critérios elaborados por Jorgenson \& Siebert (1968), apenas o modelo neoclássico manteve sua consistência interna.

A conclusão geral foi a de que os fundos internos, quando testados como determinantes do estoque de capital desejado, representavam de fato o nível do produto. Ainda, quando o produto e o fluxo de caixa eram incluídos como possíveis variáveis explicativas do estoque de capital, somente o produto predominava. Também mostraram que os fundos externos estavam subordinados às variáveis associadas ao produto. O nível do estoque de capital desejado independe das formas de financiamentos. O ranking entre os modelos foi estabelecido a partir de uma amostra de quinze grandes firmas, cujo poder de explicação do gasto de investimento foi assim classificado: 1 . neoclássico (a); 2. neoclássico (b); 3. lucros esperados; 4. acelerador; 5 . liquidez.

Elliot (1973), por sua vez, reverteu o ranking estabelecido por Jorgenson \& Siebert (1968) com uma amostra de 184 firmas, classificando segundo seus critérios, em primeiro lugar o modelo de liquidez ${ }^{4}$.

O segundo aspecto diz respeito ao set de críticas direcionadas ao modelo econométrico neoclássico ${ }^{5}$. Chirinko (1993) aponta os principais grupos de problemas de estimação. As respostas a cada um desses pontos críticos presentes na análise neoclássica motivaram a formulação de modelos de investimento denominados modelos implícito e explícito. O primeiro segue a tradição do tratamento dado por Jorgenson e seus colaboradores. $\mathrm{O}$ investimento depende da comparação entre $\mathrm{o}$ custo de uso do capital e o produto marginal do capital. O segundo grupo originou-se com Brainard \& Tobin (1968) apud Chirinko (1993) e Tobin (1969 e 1978) apud Chirinko (1993), que compara o custo de reposição de um investimento na margem para seu valor capitalizado, denominado taxa q de Tobin.

Considerando os avanços da teoria neoclássica, embora a escola tenha eclip-

\footnotetext{
${ }^{4} \mathrm{~A}$ indefinição sobre um ou outro modelo ser o mais adequado, em termos econométricos, para identificar as variáveis financiadoras do gasto de investimento, é explicada por Fazzari, Hubbard \& Petersen 1988, pp. 44-5.

${ }^{5}$ Principalmente quanto à noção de concorrência perfeita, produção previamente definida, estabilidade das expectativas dos preços relativos e da taxa de juros.
} 
sado os estudos que privilegiavam as variáveis financeiras, diante dos limites dos modelos com dinâmica implícita e explícita, as variáveis financeiras foram novamente resgatadas, porém em outras bases teóricas. A nova abordagem pressupõe que os mercados são organizados em concorrência imperfeita, para estudar o comportamento do investimento, principalmente em grupos de firmas com diferentes características financeiras. Com as imperfeições, a obtenção de fundos externos pelas firmas em muitos casos não poderia ser conseguida e, em outros, só com custos adicionais. O principal aspecto explorado do contexto da concorrência imperfeita é a assimetria de informações entre tomadores e emprestadores de recursos. Informações assimétricas impõem restrições ao funcionamento perfeito do mercado de capitais e de crédito, exigindo uma ligação entre essa hipótese e a teoria do investimento.

Com informações assimétricas, os resultados encontrados pelo modelo neoclássico podem ter uma outra interpretação, ou seja, a de que o impacto das vendas pode estar sendo subestimado, enquanto o impacto do custo do capital, superestimado. Logo, a não separação dos efeitos pode dar à abordagem neoclássica mais crédito do que lhe é devido e causar grandes danos aos agentes econômicos, se a política econômica for orientada sobre tal indeterminação. Em termos de determinação, além do benefício da separação dos elementos, como vendas do custo de capital, outras variáveis oriundas dos estudos rejeitados pelos critérios neoclássicos, testados novamente, em condições de concorrência imperfeita, puderam contribuir para o entendimento do comportamento do investimento.

\section{A HIPÓTESE DA ASSIMETRIA DE INFORMAÇÕES APLICADA À TEORIA DO INVESTIMENTO}

Fazzari, Hubbard \& Petersen (1988), Hoshi, Kashyap \& Scharfstein (1991) Fazzari \& Petersen (1993), Schaller (1993), e Hubbard, Glenn, Kashyap \& Whited (1995) testaram a importância de fatores financeiros para o gasto de investimento.

Como variáveis financeiras entende-se a estrutura financeira das firmas que determinam sua capacidade de obter fundos externos. Assim, a conexão ou interdependência entre o investimento e seu financiamento foi restabelecida por meio de verificação econométrica. Para quebrar com o teorema Modigliani \& Miller, era imprescindível mostrar que as firmas são tratadas assimetricamente pelos mercados de crédito e de capitais. Se a estrutura financeira das firmas tivesse relevância para sua condição de financiamento, estaria provada a inoperância do teorema Modigliani \& Miller e, por conseqüência, o próprio modelo de investimento neoclássico.

$\mathrm{Na}$ perspectiva de Fazzari et alli (1988), com a hipótese da assimetria de informações, os fundamentos microeconômicos que relacionam a estrutura financeira e a decisão do investimento são aqueles que justificam o elevado custo da obtenção de recursos externos para os tomadores. 


\subsection{Metodologia}

Para dar conta dos diversos estudos, suas metodologias e resultados que comprovam a hipótese da assimetria de informações, optamos por apresentar com mais detalhes duas contribuições cruciais para o entendimento da evolução contida nos modelos empíricos do investimento, construídos a partir da versão do acelerador, e que demonstraram a inconsistência do modelo neoclássico. Tratam-se dos trabalhos de Fazzari et alli (1988) e Fazzari \& Petersen (1993).

O primeiro trabalho estimulou uma série de estudos similares em diferentes países com diferentes formatos institucionais de sistemas financeiros. Foi, também, alvo de severas críticas devido à metodologia e estimação com a forma reduzida da equação do investimento. No entanto, as alternativas e melhorias realizadas em outros estudos somente fizeram confirmar os resultados obtidos por Fazzari et alli (1988). O segundo estudo, Fazzari \& Petersen (1993), porém, foi menos citado pela literatura e, do nosso ponto de vista, tem uma contribuição, da perspectiva financeira, mais adequada para se avaliar a importância da condição das firmas e sua relação com o investimento.

Considerando os modelos de liquidez contestados por Jorgenson, Fazzari et alli (1988) introduziram a novidade de investigar o comportamento do investimento a partir da influência da liquidez sobre esse gasto, utilizando um panel data com 422 firmas do setor de transformação, divididas em classes segundo o grau de retenção de dividendos com relação ao lucro a ser distribuído. Uma firma, do panel data, não é incluída na base de dados até que seja do interesse da comunidade financeira. Uma vez adicionada à base de dados, requerem-se as demonstrações financeiras dos dez anos que antecedem o ano de inclusão. Após a introdução na base de dados, a firma torna-se visível para os potenciais investidores, reduzindo então o problema da assimetria de informações, uma vez que as informações acrescentadas periodicamente permitem uma avaliação mais sistemática das condições financeiras das próprias empresas e, principalmente, de suas respectivas políticas de dividendos.

Uma firma que não distribui dividendos emite um sinal negativo ao mercado e, portanto, pode estar enfrentando problemas de liquidez ${ }^{6}$. Diante do elevado grau de retenção, os emprestadores encontram razões para negar crédito a essas firmas, associando a elas uma grande possibilidade de default. Firmas que sistematicamente distribuem dividendos não sofrem problemas de restrição à liquidez. A distribuição sistemática de dividendos garante uma avaliação positiva do mercado, mantendo ou elevando o valor da firma.

\footnotetext{
${ }^{6}$ Para evitar a presença de firmas que não tinham dividendos para distribuir, os autores mantiveram as empresas que tiveram crescimento real de vendas no período 1969-1984. Com isso excluiram-se as firmas cuja alavancagem operacional e financeira não tenha produzido o efeito sobre os lucros na dimensão necessária, permitindo então a distribuição de dividendos. Posteriormente, a inclusão de todas as firmas independentemente da evolução das vendas em termos reais não alterou de maneira significativa os resultados previamente obtidos.
} 
A estrutura inicial do modelo teve a seguinte forma:

$$
\mathrm{I} / \mathrm{Kiy}=f(\mathrm{X} / \mathrm{K}) \text { it }+g(\mathrm{CF} / \mathrm{K}) \text { it }+\mu \text { it }
$$

onde I= adição líquida de ativos fixos para a firma no período entre i e t; $\mathrm{X}$ representa o vetor de variáveis com os devidos lags, os quais têm sido destacados como os determinantes do investimento, correspondendo ao debate na literatura do investimento; $\mathrm{CF}$ indica o montante de fundos internos disponíveis para o investimento, o qual, através da função $g$ indica o grau de sensibilidade do investimento às flutuações dos fundos internos. A variável X é substituída no modelo conforme diferentes abordagens especificam a teoria do investimento, como o acelerador neoclássico e o modelo Q do investimento. Em todas as versões a variável cash flow mostrou relevância, principalmente para as firmas com baixo grau de distribuição de dividendos.

Procurando superar as dúvidas deixadas pelo trabalho de 1988, especialmente aquelas relativas ao significado da variável cash flow funcionar como uma proxy das mudanças na demanda do investimento, Fazzari \& Petersen (1993) testaram a hipótese do capital circulante líquido servir como fonte de recursos para financiar o investimento ${ }^{7}$. Embora o modelo Q tenha tido essa função, são muitas as críticas em relação à confiabilidade do modelo. A introdução do capital circulante cumpriria, assim, uma função estratégica, como veremos ${ }^{8}$.

O capital circulante é conhecido amplamente pela sua função mantenedora das atividades produtivas de curto prazo, sendo extremamente sensível às flutuações do cash flow (lucros + depreciação), e às vendas. A firma administra-o de acordo com a sua necessidade de liquidez, principalmente diante das necessidades operacionais da firma, flutuações da demanda e despesas inesperadas. A reversibilidade do capital de giro contrapõe-se à irreversibilidade do capital fixo, permitindo às firmas estabelecerem um trade-off, contraindo-o para subsidiar os gastos com investimentos fixos.

A rentabilidade das operações de uma firma determinarão a taxa de expansão desse recurso e sua resistência em épocas de crise econômica. Em princípio, a rentabilidade do capital de giro relaciona-se inversamente com seu volume, porque os recursos de curto prazo normalmente são remunerados a uma taxa menor do que os de longo prazo. Inversamente, um elevado volume de capital de giro reduziria a rentabilidade dos ativos de uma firma, dado que o capital fixo é mais rentável do que o capital circulante.

Se o parâmetro da variação do capital circulante fosse estatisticamente relevante, então os autores poderiam resolver duas questões: 1 . que o cash flow não está funcionando como uma proxy da demanda por investimento; 2 . que o capital circulante, tendo sinal negativo na equação do investimento, concorre com o

\footnotetext{
${ }^{7}$ Capital de giro é definido como a diferença entre o ativo e o passivo circulante do período corrente.

${ }^{8}$ A amostra utilizada nesse estudo contou com 48 firmas, de 1970 a 1984, com elevado grau de retenção de dividendos, e oriundas da base de dados do artigo de Fazzari et alli (1988).
} 
investimento fixo, expondo assim o grau de racionamento de crédito enfrentado pelas empresas em relação a suas classes de distribuição de dividendos. Firmas com baixo grau de distribuição deveriam depender muito mais da contração do capital circulante líquido do que firmas com alto grau de distribuição de dividendos para investir.

Mudanças no capital circulante estão positivamente relacionadas com os lucros, produto e ciclos de negócios. Então, se o capital circulante líquido funciona como uma proxy a efeitos omissos da demanda por investimento, seu coeficiente deveria ser positivo e deveria reduzir o coeficiente do cash flow, quando na equação. Porém, o coeficiente do capital circulante teve sinal negativo e, quando presente na equação do investimento, não afetou o coeficiente do cash flow.

Hoshi et alli (1991) evitaram separar as firmas pelo grau de retenção de dividendos, aspecto considerado crítico em Fazzari, Hubbard \& Petersen (1988) para identificar as empresas sujeitas ao problema da assimetria de informações. Avaliando a estrutura das relações econômicas e financeiras entre firmas e bancos no Japão, os autores beneficiaram-se de uma associação tradicional da economia japonesa, conhecida como keiretsu. Ela estabelece um vínculo bastante íntimo entre firmas e bancos, reduzindo o grau de assimetria de informações entre aqueles agentes (121 firmas) (Schaller, 1993). Firmas que não desfrutam de tal associação são denominadas de independentes (24 firmas). O objetivo dos autores foi avaliar para o Japão a importância dos recursos internos das empresas e as decisões de investimento. A estratégia dos autores foi avaliar se a liquidez é mais importante como determinante do investimento para as firmas independentes do que para firmas pertencentes à estrutura do keiretsu.

Para afastar a dubiedade acerca das variáveis de liquidez que poderiam significar o efeito de variáveis omitidas, tal como lucratividade, foi incluída nas equações a variável q de Tobin. As variáveis de liquidez foram compostas do cash flow, títulos de curto prazo rapidamente conversíveis em moeda. A conclusão dos autores é que a liquidez é mais importante para as empresas independentes do que para as associadas aos bancos$^{9}$. Assim, as primeiras são mais sensíveis às variações de sua liquidez do que as últimas.

Schaller (1993) estudou a mesma questão para o Canadá com uma amostra de 212 empresas de 1973 a 1986. De posse das experiências nos E.U.A e Japão, procurou realizar testes para contemplar as diferentes condições das firmas em fornecer informações, e seus respectivos relacionamentos com os emprestadores, especificamente no mercado de capitais, observando a capacidade das empresas de obter recursos por meio de emissão. Para o autor essa questão é primordial, uma vez que a tentativa de demonstrar a importância da liquidez das empresas para o

\footnotetext{
${ }^{9}$ Outros testes foram feitos para as empresas e também para outras formas de organização de empresas, que variavam entre associadas e independentes. Em nada alterou-se a importância das variáveis de liquidez.
} 
comportamento do investimento compreende um procedimento indireto de testar a existência das informações assimétricas ${ }^{10}$.

A composição das 212 firmas varia no estudo, de acordo com as seguintes classificações: maduras e jovens; concentradas e dispersas; do setor de transformação e de outros; associadas e independentes. Firmas maduras são aquelas presentes em um banco de dados, denominado de Laval Database, desde 1965. Firmas que não figuram desde essa data são recentes ou jovens. Com respeito à concentração, os dados vieram da Canada's Intercorporate Ownership. O grau de concentração depende de um acionista deter $50 \%$ ou mais das ações sob seu controle. De maneira análoga ao Japão, embora não exista no Canadá a organização keiretsu, existem outras formas de organização, como conglomerados, que incluem muitas firmas com ações comercializáveis em Bolsa de Valores. Muitas dessas pertencem a um indivíduo ou família. A Canada's Intercorporate Ownership informa se uma firma pertence a um conglomerado e a natureza dessa relação.

Por meio dos resultados o autor conclui que: quando as empresas são maduras, financeiramente consolidadas, com longas relações com credores, ou com elevado grau de concentração - o que permite um grau elevado de centralização das decisões, evitando portanto o conflito entre a gerência e os acionistas, a acessibilidade a fundos externos é muito favorável; quando são associadas, o problema da assimetria no mercado de capitais é menor. Empresas jovens, independentes e diversificadas enfrentam problemas de acesso ao crédito, apesar de revelarem maiores oportunidades de investimento. Isso indica o alto custo de obtenção de recursos no mercado de capitais, em oposição às firmas que, apesar de terem menos oportunidades lucrativas de investimento, são consolidadas e conseguem no mínimo três vezes mais recursos com emissão de ações.

Hubbard, Glenn, Kashayap \& Whited (1995) buscaram aplicar um método diferente para discutir a validade da assimetria de informações nos mercados financeiros e seu impacto sobre o gasto de investimento de 428 firmas entre $1976 \mathrm{e}$ 1987. A principal preocupação existente na elaboração dos modelos empíricos consiste no estabelecimento de argumentos capazes de defender a correlação entre o casf flow e o investimento como resultado das imperfeições do mercado de capitais. A verificação da correlação foi idealizada por meio da separação das empresas com relação a dois critérios: maturidade e grau de retenção de dividendos. Utilizaram as equações de Euler devido aos problemas de estimação relacionados com a medida marginal da taxa q, além das dúvidas quanto à consistência do julgamento do mercado sobre o valor da firma ${ }^{11}$.

\footnotetext{
${ }^{10}$ Embora Schaller (1993) veja mais consistência nos critérios de seleção de firmas de Hoshi et alli em relação a Fazzari et alli, deve ser observado que os arranjos institucionais que regem os mercados de capitais e de crédito impedem um relacionamento no mercado americano como aquele existente no Japão.

${ }^{11}$ As equações de Euler indicam a trajetória ótima de acumulação de capital. A firma será indiferente a aumentar o estoque de capital em uma unidade no presente e diminuir uma unidade no futuro, deixando o estoque inalterado. A firma em princípio compara os benefícios de investir no presente com
} 
Os autores estimaram os parâmetros das variáveis explicativas do investimento com as equações de Euler e em seguida discutiram porque o método não funciona adequadamente para todas as firmas da amostra. A estratégia do trabalho apóia-se em uma comparação entre um grupo de firmas para as quais a teoria neoclássica do investimento é aplicável, e um grupo para o qual a mesma teoria não o é. $\mathrm{O}$ racionamento de crédito corresponde, portanto, a uma evidência relevante somente ao grupo com a taxa baixa de distribuição de dividendos. Sendo assim, essas firmas enfrentam algum tipo de racionamento de crédito.

Estimando a trajetória ótima para as firmas que distribuem dividendos a uma taxa elevada para com o faturamento, o modelo é bem-sucedido, isto é, o comportamento é como descrito pela teoria neoclássica. Para as firmas que distribuem poucos dividendos em relação ao faturamento, o modelo é rejeitado. O modelo é também rejeitado para a amostra total. Os autores também não encontraram maior relevância para estimar o modelo em função do tamanho das firmas.

O insucesso do modelo para as firmas com baixo grau de distribuição de dividendos pode não significar o efeito das imperfeições dos mercados de capitais. Para verificar a procedência do argumento, os autores testaram o modelo para firmas maduras e com baixo grau de distribuição de dividendos. A importância do volume do cash flow para essas firmas poderia ser uma decisão da gerência em aproveitar oportunidades de investimentos. Segundo as previsões neoclássicas, o modelo responde para as firmas maduras com baixo grau de distribuição de dividendos. Porém, o custo do crédito segundo a disponibilidade do cash flow e a medida agregada de aperto de liquidez afeta o gasto de investimento das firmas para as quais o modelo neoclássico foi rejeitado, permitindo aos autores concluir que as imperfeições do mercado de capitais afetam as decisões de investimento.

\section{RESULTADOS E OBSERVAÇÕES FINAIS}

O conjunto de estudos empíricos, com relativa diversidade metodológica, indica claramente a refutação das predições neoclássicas, seja a partir de uma versão do acelerador, seja por meio das equações de Euler. Com as equações de Euler não é possível verificar as diferentes condições financeiras das firmas na obtenção de recursos. O estudo com equação reduzida do investimento é mais apropriada porque permite estabelecer políticas específicas, uma vez identificadas as fontes de financiamento do investimento das firmas.

A tabela abaixo sintetiza os principais resultados (valor dos parâmetros das variáveis testadas na equação de investimento) dos estudos resenhados que impuseram diferentes critérios de separação de firmas de diferentes amostras, sujeitas à assimetria de informações.

o investimento no futuro. A diferença entre a produtividade marginal do capital e seu custo impele ao investimento. 


\begin{tabular}{|c|c|c|c|c|c|c|c|}
\hline \multirow{2}{*}{$\begin{array}{c}\text { Variáveis } \\
\text { Indepen- } \\
\text { dentes }\end{array}$} & \multicolumn{2}{|c|}{$\mathrm{FHP}^{*}(1988)$} & \multirow[t]{2}{*}{$\begin{array}{c}\mathrm{FP}^{* *} \\
\text { (1993) }\end{array}$} & \multicolumn{2}{|c|}{$H K S * * *(1991)$} & \multicolumn{2}{|c|}{ SCHALLER $* * *(1993)$} \\
\hline & $\begin{array}{c}\text { Neo- } \\
\text { clássico }\end{array}$ & Acelerador & & $\begin{array}{l}\text { Indepen- } \\
\text { dentes }\end{array}$ & Associadas & $\begin{array}{l}\text { Indepen- } \\
\text { dentes }\end{array}$ & Associadas \\
\hline $\mathrm{CF}$ & 0.319 & 0.286 & 0.743 & 0.501 & 0.060 & 0.36 & 0.37 \\
\hline$J$ & 0.275 & & & & & & \\
\hline O & 0.0005 & -0.0004 & 0.0054 & 0.003 & 0.011 & 1.55 & -0.13 \\
\hline$S$ & & 0.042 & & & & & \\
\hline VCG & & & -430 & & & & \\
\hline
\end{tabular}

CF = cash flow; $\mathrm{J}=$ Parâmetro de ajustamento entre o estoque de capital desejado e as vendas ajustadas aos preços relativos do capital; $\mathrm{Q}=$ modelos com taxa q; $\mathrm{S}=$ vendas; VCG = variação do capital circulante líquido.

*Fazzari et alli, (1988). **Fazzari \& Petersen (1993). ${ }^{* * * H o s h i ~ e t ~ a l l i, ~(1991) . ~}{ }^{* * *}$ Schaller (1993).

A despeito das diferenças entre os sistemas financeiros dos estudos, verificase, de maneira geral, a existência de racionamento de crédito. As evidências confirmam a importância dos fundos internos como fonte de financiamento e a hipótese da assimetria de informações. O comportamento do investimento no âmbito da firma é melhor explicado quando é incorporado à variável cash flow, deslocando a importância da teoria neoclássica. Por fim, observamos que, apesar de a metodologia de Fazzari et alli (1988) ter servido de base para outros estudos, aqueles que usaram critérios qualitativos para separar as firmas demonstram-se mais consistentes. Especificamente, a separação meramente quantitativa, como a feita em função do grau de retenção de dividendos, tem sido duramente criticada ${ }^{12}$. A não-observância das características do formato institucional dos sistemas financeiros, como feitos por Hoshi et alli (1991) e Schaller (1993) pode induzir a uma falsa percepção sobre as restrições creditícias e o comportamento do investimento.

\section{REFERÊNCIAS BIBLIOGRÁFICAS}

ANDERSON, W. H. L. Corporate Finance and Fixed Investment: An Econometric Study. Boston: Division of Research, Graduate School of Bussiness Administration, Harvard University, 1964.

BRESSER-PEREIRA, L. C. Interest rate and investment decision in normal and excepcional times. Trabalho apresentado em conferência em Knoxville, Tennessee, julho de 1993.

CHIRINKO, R. S. Business fixed investment spending: modeling strategies, empirical results, and policy implications. Journal of Economic Literature, 1993, v. 31, pp. 1875-1911.

EISNER, R. "Investment: Fact and Fancy.” American Economic Review, 1963, v. 53, pp. 237-46.

ELLIOT, W. "Theories of corporate investment behavior revisited." American Economic Review, 1973, v. 63 , p. $195-207$.

EVANS, M. “A Study of Industry Investment Decisions." Review of Economics and Statistics, 1967, v. 49, pp. 151-64.

FAZZARI, S. M., HUBBARD, G. \& PETERSEN, B. Financing constraints and corporate investment. Brookings Papers on Economic Activity, 1988, n. 1, pp. 141-95.

${ }^{12}$ Ver Kaplan \& Zingales (2000). 
FAZZARI, S. \& PETERSEN, B. Working capital and fixed investment: new evidence on finance constraints. Randal Journal of Economics, 1993, v. 24, n. 3, pp. 328-42.

HALL, R. \& JORGENSON, D. Tax policy and investment behavior. American Economic Review, 1967 , v. 57, pp. 391-414.

HOSHI, T., KASHYAP, A. K., \& SCHARFSTEIN, D. Corporate structure, liquidity, and investment: evidence from Japanese industrial groups. Quarterly Journal of Economics, 1991, v. 106, pp. 33 60.

HUBBARD, R., GLENN, A. KASHYAP, \& WHITED, T. M. Internal Finance and Firm Investment. Journal of Money, Credit, and Banking, 1995, v. 27, n. 3, pp. 683-701.

JORGENSON, D. W. \& SIEBERT, C. D. A comparison of alternative theories of corporate investment behavoir. The American Economic Review, 1968, v. 58, n. 4, pp. 681-712.

JORGENSON, D. Econometric studies of investment behavior: a survey. Journal of Economic Literature, 1971, v. 9, n. 4, pp. 1111-46.

MEYER, J. \& KUH, E. The Investment Decision: An Empirical Study. Cambridge, Mass.: Harvard University Press, 1957.

MEYER, J. \& GLAUBER, R. Investment Decisions, Economic Forecasting and Public Policy. Boston: Division of Research, Graduate School of Bussiness Administration, Harvard University, 1964.

PINDYCK, R. Irreversibility, uncertainty and investment. Journal of Economic Literature, 1991, v. 29, n. 3, pp. 1110-48.

KAPLAN, S. \& ZINGALES, L. Investment - cash flow sensitivities are not valid measures of financing constraints. NBER, working paper, n. 7659.

SCHALLER, H. “Asymmetric Information, Liquidity Constrains, and Canadian Investment.” Canadian Journal of Economics, 1993, v. 26, n. 3, pp. 542-73. 